\title{
Dentatorubral pallidoluysian atrophy
}

INSERM

\section{Source}

INSERM. (1999). Orphanet: an online rare disease and orphan drug data base.

Dentatorubral pallidoluysian atrophy. ORPHA:101

Dentatorubral pallidoluysian atrophy (DRPLA) is a rare subtype of type I autosomal dominant cerebellar ataxia (ADCA type I; see this term). It is characterized by involuntary movements, ataxia, epilepsy, mental disorders, cog nitive decline and prominent anticipation. 